\title{
Ablating the renal nerves to treat heart failure with reduced ejection fraction: another treatment option in a crowded space?
}

\author{
Murray Esler ${ }^{1}$
}

Received: 29 October 2020 / Accepted: 30 October 2020 / Published online: 11 November 2020

c) Springer-Verlag GmbH Germany, part of Springer Nature 2020

Keywords Renal denervation $\cdot$ Sympathetic nervous system $\cdot$ Heart failure $\cdot$ Renal sympathetic nerves

Howard Levin and Mark Gelfand first showed the feasibility of catheter-based renal denervation for cardiovascular diseases almost 20 years ago [US provisional patents 60/370 190 (April 2002), 60/415 575 (October 2002) and 60/442 970 (January 2003)]. Sympathetic nerves pass to the human kidneys via the outer wall and peri-adventitial region of the renal arteries, within reach of ablative energy delivered from the artery lumen. The renal sympathetic outflow is activated in hypertension, heart failure, obesity and hepatic cirrhosis [1], so that the renal sympathetic nerves came to be seen as a logical, potential target in these disorders. Levin and Gelfand initially envisioned an application of catheter-based renal denervation in heart failure.

The patents for renal denervation were acquired by The Foundry, an innovative venture capital group, which initiated a program of renal denervation in severe, drug-resistant hypertension. This was conducted by the start-up company, Ardian, whose engineers developed and tested renal denervation catheters delivering radiofrequency energy. The first two trials of catheter-based renal denervation for drugresistant hypertension commenced in Melbourne in 2007 and 2009, with subsequent publication of the trial outcomes in the Lancet in 2009 and 2010 [2, 3]. Renal denervation is now an approved device for the treatment of hypertension in more than 20 countries, in spite of which it remains experimental in US. In this issue of Clinical Autonomic Research, a meta-analysis by Lian and colleagues [4] takes renal denervation back full circle to its origins, the planned therapeutic application of this technology in heart failure.

The meta-analysis is timely. A previous review of renal denervation in heart failure suggested benefit, but was based

Murray Esler

murray.esler@baker.edu.au

1 Baker Heart and Diabetes Institute, PO Box 6492, Melbourne, VIC 3004, Australia on too few studies to be definitive [5]. The addition of six original studies published in the past 3 years now allows the authors of this meta-analysis to conclude that the treatment is both safe and beneficial. The meta-analysis conformed to the features expected for validity. The Preferred Reporting Items for Systematic Reviews and Meta-Analyses (PRISMA) guidelines were followed [6]. Strict and appropriate trial inclusion and exclusion criteria were applied. The Newcastle-Ottawa Scale was used to assess the quality of the trials (https://www.ohri.ca/clinical_epidemiology/oxfor d.asp). The contribution of each trial to the pooled estimates of effects was assessed in a sensitivity analysis. Publication bias was tested for with Begg's funnel plot and Egger's test. Eleven studies passed muster and were included.

The meta-analysis demonstrated benefit of renal denervation in heart failure with reduced ejection fraction. The analysis did not allow comparison of risks/benefits for renal nerve ablation achieved with radiofrequency energy versus ultrasonic energy. Demonstrated benefits from renal denervation were an increase in left ventricular ejection fraction, reductions in left-ventricular end-systolic diameter, leftventricular end-diastolic diameter, and left atrial diameter, and a fall in the plasma concentration of B-type natriuretic peptide [4]. As the treatment is not reversible, the demonstrated safety out to 12 months of follow-up post-procedure is important. There was no fall in blood pressure. This was perhaps surprising, as blood pressure reduction is the endpoint sought and often achieved in the commonest application of renal denervation, which is, resistant hypertension.

Consideration of the neural pathophysiology of cardiac failure with reduced ejection fraction is helpful, in providing the theoretical backdrop to renal denervation treatment. Anti-adrenergic therapy until now has primarily involved $\beta$-adrenergic blockade which counters the high level of activation of the cardiac sympathetic outflow, a proven factor in heart failure lethality [7]. The sympathetic outflow to the 
kidneys is also activated at an extreme level in patients with cardiac failure [1], having the adverse effects of reduction in renal blood flow, stimulation of renal secretion, and renal tubular reabsorption of sodium; the renal tubules have dense sympathetic innervation [8]. In a prior study in heart failure patients [9], the level of activation in the renal sympathetic outflow, measured with renal noradrenaline spill over methodology, was a strong, direct predictor of reduced survival. It is this renal neural pathophysiology in heart failure which is now targeted head-on with renal denervation. In addition, the renal denervation procedure ablates renal afferent nerves which contribute to central sympathetic outflow, lowering generalized sympathetic activity [10].

Despite the positives conveyed by the meta-analysis of Lian et al. [4], there remain two hurdles to jump before renal denervation can become an established therapy for heart failure with reduced ejection fraction: the first is that proving a treatment actually prolongs survival in heart failure is crucial. In the past, surrogate hemodynamic endpoints have been disastrously misleading. Cardiac inotropes improved intra-cardiac hemodynamics in the short term, but contributed to ventricular arrhythmias and early death. To perform studies of sufficient scale to test for benefit of RDN on survival will be daunting. Perhaps the regulators will not request this, but doing that would break with current practice in the evaluation of drugs tested in heart failure.

The second hurdle concerns the fact that the pharmacological treatment of heart failure has become a "crowded space". Drugs of proven benefit are ACE-inhibitors, $\beta$-adrenergic blockers, angiotensin receptor-blockers, mineralocorticoid antagonists, neprilysin inhibitor-ARB combinations (ARNIs) and SGLT2 inhibitors. Using these drugs in combination takes finesse. They are never all used concurrently. With use of these drugs in combination, a fall in blood pressure can become the limiting factor. Where might renal denervation fit in? That will be the question to answer after regulatory approval is given. The fact that renal denervation does not lower blood pressure in patients with heart failure will help. Will it be rational to combine renal denervation with anti-adrenergic drugs? Perhaps not with beta-blockers, but renal denervation may be acceptable with a mineralocorticoid antagonist or SGLT2 inhibitor, which possibly have anti-adrenergic activity. It could be that we are straying too far into the future. Certainly, any suggestion that renal denervation might now be used in heart failure with preserved ejection fraction would be straying too far.

Acknowledgements Supported by a Victorian State Government Infrastructure Support Grant to the Baker Heart and Diabetes Institute.

\section{Compliance with ethical standards}

Conflict of interest Prof. Esler is a member of the Renal Denervation Medical Advisory Boards of SyMap (China) and Medtronic (US).

\section{References}

1. Esler M, Jennings G, Korner P, Willett I, Dudley F, Hasking G et al (1988) The assessment of human sympathetic nervous system activity from measurements of norepinephrine turnover. Hypertension 11:3-20

2. Krum H, Schlaich M, Whitbourn R, Sobotka P, Sadowski J, Bartus $\mathrm{K}$ et al (2009) Catheter-based renal sympathetic denervation for resistant hypertension: a multicentre safety and proof-of-principle cohort study. Lancet 373:1275-1281

3. Symplicity HTN-2 Investigators (CI Esler M) (2010) Renal sympathetic denervation in patients with treatment-resistant hypertension (The Symplicity HTN-2 Trial): a randomized controlled trial. Lancet 376:1903-1909

4. Lian Z, Shi-Ran Yu, Song J-X, Lee C-Y, Li S-F, Cui Y-X et al (2020) Efficacy and safety of catheter-based renal denervation for heart failure with reduced ejection fraction: a systematic review. Clin Autonom Res. https://doi.org/10.1007/s10286-020-00716-y

5. Fukuta H, Goto T, Wakami K, Ohte N (2017) Effects of catheterbased renal denervation on heart failure with reduced ejection fraction: a systematic review and meta-analysis. Heart Fail Rev 22:657-664

6. Moher D, Liberati A, Tetzlaff J (2009) Preferred reporting items for systematic reviews and meta-analyses: the PRISMA statement. Ann Intern Rev 151:264-269

7. Kaye DM, Lefkovits J, Jennings GL, Bergin P, Broughton A, Esler MD (1995) Adverse consequences of high sympathetic nervous activity in the failing human heart. J Am Coll Cardiol 26:1257-1263

8. Di Bona GF (2000) Nervous kidney. Interaction between renal sympathetic nerves and the renin-angiotensin system in the control of renal function. Hypertension 36:1083-1088

9. Petersson M, Friberg P, Eisenhofer G, Lambert G, Rundqvist B (2005) Long-term outcome in relation to renal sympathetic activity in patients with chronic heart failure. Eur Heart J 26:906-913

10. Hering D, Lambert EA, Marusic P, Walton AS, Krum H, Lambert GW et al (2013) Substantial reduction in single sympathetic nerve firing after renal denervation in patients with resistant hypertension. Hypertension 61:457-464 